\title{
Resuming In-Person Psychotherapy Sessions with Perinatal Women amid the COVID-19 Pandemic: A Multistakeholder Perspective
}

Nicole Andrejek

Lunenfeld-Tanenbaum Research Institute

Sabrina Hossain

Lunenfeld-Tanenbaum Research Institute

Nour Schoueri-Mychasiw

Lunenfeld-Tanenbaum Research Institute

Gul Saeed

University of Toronto

Maral Zibaman

Lunenfeld-Tanenbaum Research Institute

Angie Puerto Niño

Lunenfeld-Tanenbaum Research Institute

Samantha Meltzer-Brody

University of North Carolina at Chapel Hill School of Medicine

Richard Silver

NorthShore University HealthSystem

Simone N. Vigod

Women's College Hospital

Daisy Radha Singla ( $\nabla$ daisy.singla@utoronto.ca )

Lunenfeld-Tanenbaum Research Institute https://orcid.org/0000-0001-9865-7112

\section{Research}

Keywords: COVID-19, perinatal depression, perinatal anxiety, psychotherapy, barriers, facilitators, inperson healthcare

Posted Date: July 20th, 2021

DOI: https://doi.org/10.21203/rs.3.rs-675643/v1

License: (c) (1) This work is licensed under a Creative Commons Attribution 4.0 International License.

Read Full License 


\section{Abstract}

BACKGROUND: During the COVID-19 pandemic, essentially all outpatient psychotherapy with perinatal patients transitioned to telemedicine. The aim of this study was to examine how to resume in-person

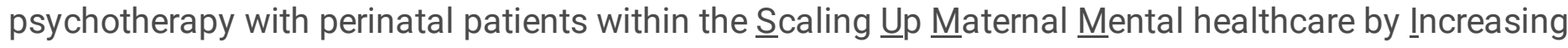
access to Ireatment (SUMMIT) trial as the pandemic abates.

METHODS: We conducted focus groups and individual interviews with perinatal participants $(n=23)$, providers $(n=28)$, and a range of stakeholders $(n=18)$ who participated in the SUMMIT trial from August 2020 to March 2021. Content analysis was used to examine (1) previous barriers that impacted perinatal participants access or decision to access in-person healthcare during COVID-19; (2) perceived future barriers for perinatal participants to attend in-person sessions; and (3) potential facilitators to resuming in-person sessions with perinatal participants.

RESULTS: Perinatal participants (69.6\%) and providers (57.1\%) reported that COVID-19 previously impacted perinatal SUMMIT patients' access or decision to access in-person healthcare. Specifically, "external barriers" such as institutional regulations due to COVID-19 (100\% perinatal participants and $68.8 \%$ providers) and "internal barriers" such as concerns about being exposed to COVID-19 (75.0\% perinatal participants and $56.3 \%$ providers) impacted perinatal participants access to in-person care. Perceived barriers to resuming in-person psychotherapy sessions included concerns about virus exposure in a hospital setting (77.8\% stakeholders, $73.9 \%$ perinatal participants, $71.4 \%$ providers); virus exposure while taking public transportation ( $50.0 \%$ stakeholders, $26.1 \%$ perinatal participants, $25.0 \%$ providers). Stakeholders (50.0\%) were more likely than providers $(25.0 \%)$ and participants $(13.0 \%)$ to endorse wearing a mask during sessions as a barrier. Key facilitators to resuming in-person psychotherapy sessions were the implementation and effective communication of robust safety protocols $(72.2 \%$ stakeholders, $47.8 \%$ perinatal participants, $39.3 \%$ providers) and providing alternative locations to where sessions are delivered, e.g., using large rooms ( $44.4 \%$ stakeholders, $32.1 \%$ providers, $17.4 \%$ perinatal participants).

CONCLUSION: The results suggest that a significant majority of perinatal participants experienced barriers to accessing in-person healthcare during the pandemic, and concerns about contracting the virus within and en route to the hospital remain while the COVID-19 pandemic persists. To overcome these barriers, our study suggests that implementing and effectively communicating robust safety protocols may be the most effective methods to address these concerns when resuming in-person psychotherapy sessions.

\section{Contributions To The Literature}

- We provide insights into relevant barriers and facilitators among perinatal women with symptoms of depression and anxiety to participate in in-person psychotherapy amid the COVID-19 pandemic. 
- Although we found that that the COVID-19 pandemic has impacted perinatal women access to inperson healthcare, and there may be ongoing barriers for perinatal women to resume in-person psychotherapy sessions during the pandemic, implementing and clearly communicate robust safety protocols to study participants and conducting psychotherapy not deep within the hospital setting or in larger-sized rooms are two ways to facilitate resuming in-person sessions.

- Our findings contribute valuable knowledge on best practices and procedures for clinical practices and research to resume in-person activities amid COVID-19.

\section{Background}

The COVID-19 pandemic has exacerbated depression and anxiety symptoms among perinatal populations [, ]. During the pandemic, many medical interventions-including in-person psychotherapy sessions-were postponed, stopped, or altered to avoid in-person contact among staff and patients [, ]. To continue providing care, in-person psychotherapy sessions quickly transitioned to telemedicine [ ]. Although there is a growing body of evidence that suggests that telemedicine is a convenient [, efficacious [, and an acceptable mode of receiving psychotherapy [, there remains a need for further evidence to decipher whether psychotherapy delivered via telemedicine is as effective as receiving it inperson [, ], and for whom telemedicine may be most suitable []. In order to answer this important question, it is critical to understand relevant barriers and facilitators to optimize the implementation of in-person psychotherapy.

Recent studies suggest that approximately 8 to $13 \%$ of women experience perinatal depression in Canada and the United States [ ], and 15-20\% of women experience perinatal anxiety [. Psychotherapy has been shown to be effective in reducing and preventing the significant burden of perinatal depression and anxiety [, ]; however, as few as $12 \%$ of women with perinatal depression access mental healthcare services $[$. When considering the potential barriers to resuming in-person psychotherapy with perinatal women during the COVID-19 pandemic, this population may be reluctant to attend in-person sessions because they may feel particularly vulnerable and fearful of contracting COVID-19 while pregnant or exposing their newborn [.

In this study we explored the factors that might impact perinatal participants' decision to attend in-person psychotherapy sessions during COVID-19 from a multistakeholder perspective within the context of the Scaling Up Maternal Mental healthcare by Increasing access to Treatment (SUMMIT) trial D. SUMMIT is a randomized clinical trial aimed at increasing access to mental health treatment among perinatal women with symptoms of depression and anxiety in Canada and the United States by delivering a form of psychotherapy called behavioral activation (BA). Though a central aim of the larger trial is to compare telemedicine to in-person, all SUMMIT participants were randomized to telemedicine beginning in midMarch 2020 due to COVID-19. With the recent rollout of COVID-19 vaccines and a reduction in cases in some jurisdictions, it is critical to examine how to safely resume in-person psychotherapy with perinatal women within the SUMMIT trial. 
From an implementation science framework [ ], it is worthwhile to identify and explore potential barriers and facilitators $\square$ when examining how to resuming in-person psychotherapy to inform best practices that can be applied to SUMMIT, as well as other research and healthcare activities aiming to resume in-person activities during COVID-19 [22]. The current study therefore included three objectives: (1) to examine whether COVID-19 had previously impacted perinatal participants' access or decision to access in-person healthcare; (2) to identify potential future COVID-19-specific barriers that might impact perinatal participants' decision to participate in in-person psychotherapy as the pandemic persists; and (3) to explore potential facilitators to resuming in-person psychotherapy sessions with future perinatal participants within the SUMMIT trial. In this study, our analysis focuses exclusively on previous and future barriers to accessing in-person psychotherapy that are specific to the COVID-19 pandemic, rather than barriers that would likely exist for perinatal participants regardless of the pandemic, such as a lack of childcare or convenience. We drew on the perspectives of perinatal participants who received BA and the providers who delivered BA within the SUMMIT trial. We also drew on the diverse perspectives from a range of community and hospital-based stakeholders.

\section{Methods}

\section{Study Sites and Ethics}

SUMMIT, a multi-site, four-arm, randomized, non-inferiority trial, is being conducted across three hubs in Toronto, Canada; and in Chapel Hill and Chicago in the United States. Ethical approval was obtained from Clinical Trials Ontario Research Ethics Board [1895], Institutional Review Board of NorthShore University HealthSystems [EH18-129], and UNC Biomedical Institution Review Board [19-1786]. Full details of the SUMMIT trial protocol have been published elsewhere $\left[{ }^{20}\right]$.

\section{Sample}

The current study includes data from three types of participants: perinatal participants, provider participants, and stakeholder participants (see below).

\section{Perinatal Participants}

We selected perinatal participants for interviews from the larger SUMMIT trial, which recruits women who are 18 years of age or older and are either pregnant (up to 36 weeks) or postpartum (4 to 30 weeks) with depressive symptoms (EPDS $\geq 10$ ). Exclusion criteria for the larger trial were: non-English or non-Spanish speakers; women with active suicidal intent, active symptoms of psychosis or mania, and/or active substance abuse or dependence; women whose psychotropic medication dose or medication changed within 2 weeks of enrolment or beginning the BA sessions; women who were in ongoing psychotherapy; and women who experienced severe fetal anomalies; stillbirth, or infant death at the time of enrolment for index pregnancy [20]. To be eligible to participate in the current study, perinatal participants must have consented to participate in qualitative interviews during the original SUMMIT consent process and must 
have completed at least five BA sessions. Among consenting perinatal participants, a randomly selected subset of all eligible perinatal participants trial were recruited. Site-specific research teams contacted these participants via email, phone calls, or text messages.

\section{Provider Participants}

The provider group consisted of non-specialist providers (NSPS) and specialist providers (SPs) who delivered the BA sessions in the SUMMIT trial. NSPs were nurses and midwives with no previous mental health expertise that received training to deliver BA. SPs were psychologists, psychiatrists, and social workers. To be eligible to participate in the current study, providers must have consented to participate in qualitative research. SUMMIT's qualitative research team used convenience sampling and invited them to participate in this study via email, phone calls, or text message.

\section{Stakeholder Participants}

The stakeholder group comprised of community-based stakeholders, including patient advocates, clinicians, community partners, and study consultants, and hospital-based stakeholders, which included psychiatrists, hospital administrators, and clinicians located at SUMMIT's associated sites. Stakeholders were contacts of the diverse SUMMIT team across study sites and were recruited by convenience sampling. Once recruited, the qualitative research team invited them to participate in this study via email.

\section{Data Collection}

Data collection took place between August 2020 and March 2021. We conducted semi-structured individual and focus group interviews to examine barriers and facilitators to resuming in-person psychotherapy sessions within the SUMMIT trial that were specific to the COVID-19 pandemic. Specifically, we asked perinatal participants and providers whether COVID-19 previously impacted SUMMIT perinatal participants' access or decision to access in-person healthcare and what those barriers specifically were to learn about potential barriers SUMMIT might also encounter. We then asked all participants their perspectives on what they foresee might be future barriers to resuming in-person psychotherapy sessions with perinatal women that are specific to the COVID-19 pandemic. Last, we asked all participants their perspectives about potential facilitators to resuming in-person psychotherapy sessions with perinatal women amid the pandemic (see Appendix A for sample interview questions).

Two trained qualitative researchers (authors NA and SH) conducted all individual and focus group interviews following the HIPPA/PHIPA-compliant version of Zoom ${ }^{\mathrm{T}}$. Both interviewers identify as female. The research team conducted 8 focus group interviews with providers, with a range of two to six providers in each, and one focus group interview with nine stakeholders. Three individual interviews were conducted with providers who were unable to attend the focus groups and nine individual interviews were conducted with stakeholders who were eligible for interviews later on in the data collection process. We also conducted individual interviews with perinatal participants to maintain confidentiality, capture their unique personal experiences in the SUMMIT trial, and explore their perspectives on attending in-person psychotherapy during COVID-19. Interviews were conducted until saturation was reached. Participants 
either signed a paper consent form or an online consent form via REDCap ${ }^{\mathrm{TM}}$. The researchers obtained both written and verbal consent before conducting all interviews and gave full details of the study prior to beginning interviews. Interviews and focus groups lasted approximately 40 to 60 minutes. All participants received an e-gift card (\$15 USD or \$20 CAD) as remuneration for their participation in the interviews. All participants were assigned an identification code and any identifying details were obscured to maintain confidentiality.

\section{Data Analysis}

Trained research assistants audio-recorded the interviews, encrypted them for security, transcribed verbatim, and checked their quality. Qualitative data were analyzed using NVivo ${ }^{\mathrm{TM}}$ by authors $\mathrm{SH}$ and NA. Using previously established methodologies [23, ], we conducted a content analysis $\square$ to identify common barriers and facilitators to resuming in-person sessions. We calculated inter-rater reliability using the Kappa ( $\mathrm{k}$ ) coefficient $(\mathrm{K}=0.95)$, indicating a substantial agreement between the authors (NA and $\mathrm{SH})$. We used an iterative coding process that identified patterns across participants' responses through two stages of coding: an initial coding stage and a secondary, focused coding stage. In addition, we calculated descriptive results (means, ranges, and frequencies) for relevant demographic characteristics. We derived perinatal participant and provider descriptive data from baseline questionnaires that were completed for the larger SUMMIT trial prior to participating in interviews (see Appendix B for the baseline questions). Stakeholder demographic data were not collected as a part of the larger SUMMIT trial.

\section{Results}

Our results describe the qualitative data collected from perinatal participants participating in the larger SUMMIT trial $(n=23)$, providers delivering BA in the trial $(n=28)$, and a range of stakeholders $(n=18)$, across Canada and the United States (see Table 1 for participant characteristics). 
Table 1

Participant demographic characteristics

\section{Perinatal participants $(n=23)$ \\ Frequency (\%) unless otherwise indicated}

Age

Mean and range

$32.0(20-40)$

Location

Canada

$14(60.9)$

United States

$9(39.1)$

Race/ethnicity

White

$12(52.2)$

Other

$9(39.1)$

Prefer not to answer

2 (8.7)

Marital Status

Married or stable relationship

19 (82.6)

Single or dating

$5(21.7)$

Prefer not to answer

2 (8.7)

Employment

Maternity Leave

$8(34.8)$

Full-time employment

$6(26.1)$

Part-time employment

$3(13.0)$

Unemployed

$3(13.0)$

Other

$3(13.0)$

Highest Level of Education

High School or College/Trade School

$5(21.7)$

University (undergraduate degree)

$8(34.8)$

University (graduate degree)

$10(43.5)$

Household income

$\$ 0-\$ 39,999$

$4(1)$

$\$ 40,000-\$ 79,999$

4 (17.4) 


\begin{tabular}{|c|c|}
\hline Perinatal participants $(n=23)$ & $\begin{array}{l}\text { Frequency (\%) unless otherwise } \\
\text { indicated }\end{array}$ \\
\hline$\$ 80,000$ or more & $13(56.5)$ \\
\hline Prefer not to answer & $2(8.7)$ \\
\hline \multicolumn{2}{|l|}{ Number of children } \\
\hline No children, pregnant & $8(34.8)$ \\
\hline 1 child & $3(13.0)$ \\
\hline 2 children & $4(17.4)$ \\
\hline Providers $(n=28)$ & $\begin{array}{l}\text { Frequency (\%) unless otherwise } \\
\text { indicated }\end{array}$ \\
\hline \multicolumn{2}{|l|}{ Age } \\
\hline Mean and range & 44 (41.3 to 46.6$)$ \\
\hline \multicolumn{2}{|l|}{ Location } \\
\hline Canada & $12(42.9)$ \\
\hline United States & $16(57.1)$ \\
\hline \multicolumn{2}{|l|}{ Provider Type } \\
\hline Specialists providers (SP) & $13(46.4)$ \\
\hline Non-specialists providers (NSP) & $15(53.6)$ \\
\hline \multicolumn{2}{|l|}{ Gender } \\
\hline Female & $26(92.9)$ \\
\hline Male & $2(7.1)$ \\
\hline Stakeholders $(n=18)$ & $\begin{array}{l}\text { Frequency (\%) unless otherwise } \\
\text { indicated }\end{array}$ \\
\hline \multicolumn{2}{|l|}{ Location } \\
\hline Canada & $9(50.0)$ \\
\hline United States & $9(50.0)$ \\
\hline \multicolumn{2}{|l|}{ Stakeholder Type } \\
\hline $\begin{array}{l}\text { Community-based (patient advocates, clinicians, community } \\
\text { partners) }\end{array}$ & $9(50.0)$ \\
\hline $\begin{array}{l}\text { Hospital-based (psychiatrists, hospital administrators, and } \\
\text { clinicians) }\end{array}$ & $9(50.0)$ \\
\hline
\end{tabular}




\section{Objective 1. Accessing healthcare during COVID-19}

When asked whether COVID-19 impacted perinatal participants' access or decisions to access their regular healthcare (such as going to their family doctor), the majority of perinatal participants ( $n=16$ of $23,69.6 \%)$ stated that COVID-19 had impacted their access. This finding was affirmed by a majority of providers ( $n=16$ of $28,57.1 \%$ ), who stated that they were aware that their SUMMIT patients experienced barriers to accessing their regular healthcare through their discussions of their patients' health, wellbeing, and anxieties during the BA sessions. Thus, they had relevant insights about the impacts of COVID-19 on their participants' access to healthcare.

Of the 16 perinatal participants and 16 providers who reported that COVID-19 impacted their or their SUMMIT patients' access to healthcare respectively, two types of barriers emerged (see Table 2 for an overview of the barriers): (1) external barriers, which referred to institutional or government regulations that impacted healthcare service delivery (perinatal participants: $n=16$ of $16,100 \%$; providers: $n=11$ of $16,68.8 \%)$; and (2) internal barriers, which referred to barriers derived from personal choices, anxieties, or fears over the potential for virus exposure (perinatal participants: $n=12$ of $16,75.0 \%$; providers: $n=9$ of $16,56.3 \%)$.

Table 2

Reported barriers to accessing healthcare during COVID-19, n (\%)

\begin{tabular}{|c|c|c|}
\hline Key Themes & $\begin{array}{l}\text { Perinatal } \\
\text { participants } \\
(\mathrm{n}=23)\end{array}$ & $\begin{array}{l}\text { Provider } \\
\text { Participants } \\
(\mathrm{n}=28)\end{array}$ \\
\hline Yes, barriers were experienced & $16(69.6)$ & $16(57.1)$ \\
\hline $\begin{array}{l}\text { Of the participants who said "yes", the barriers addressed were } \\
\text { "external barriers" (e.g., institutional or governmental regulations) }\end{array}$ & $16(100.0)$ & $\begin{array}{l}11 \text { of } 16 \\
(68.8)\end{array}$ \\
\hline $\begin{array}{l}\text { Of the participants who said "yes", the barriers addressed were } \\
\text { "internal barriers" (e.g., personal choices, anxieties, or fears over the } \\
\text { potential for virus exposure) }\end{array}$ & $12(75.0)$ & $\begin{array}{l}9 \text { of } 16 \\
(56.3)\end{array}$ \\
\hline No Barriers & $6(26.1)$ & $8(28.6)$ \\
\hline Unsure/No response & $1(4.3)$ & $4(14.3)$ \\
\hline
\end{tabular}

External barriers to accessing healthcare during COVID-19

Of the 16 perinatal participants who stated that COVID-19 impacted their access to healthcare, all 16 (100.0\%) reported experiencing external barriers. Similarly, 11 (68.8\%) providers reported that external barriers impacted their BA patients' access to care, with one who expressed that:

"I see messages all the time... [about] what [patients'] visits are going to look like [due to COVID-19 related guidelines]... I think it's been hard for patients to figure out" (SP_04_US). 
Perinatal participants also described these new regulations during COVID-19 could be stressful and challenging:

"I can never get [a hold of my family doctor]... So that's been [difficult]... My first appointment [during COVID] took a few weeks [to get] and it was really anxiety inducing because it's my first pregnancy... All aspects from my home doctor, to midwifery, to my OB; COVID definitely impacted me." (Perinatal_16_ Canada)

"Breastfeeding clinics were closed so that was hard...I know they were offering things online, but I actually had trouble breastfeeding and didn't access [help] because [finding an online service was] tough. I needed hands-on help, and I thought: 'no way I could get enough support online'." (Perinatal_24_ Canada)

Internal barriers in accessing healthcare during COVID-19

Of the 16 perinatal participants who stated that they had experienced barriers to their usual healthcare, three-quarters (12 of $16 ; 75.0 \%$ ) explained that internal barriers impacted their decisions to access healthcare. Likewise, of the 16 providers who stated that their participants reported experiencing barriers to their usual healthcare, 9 (56.3\%) stated that their SUMMIT patients experienced internal barriers to accessing healthcare. A participant explained that:

"In my first trimester, I was very sick, very dehydrated. I needed fluids, probably pretty bad where I should have gone to the ER but I chose not to because I didn't want to get exposed during COVID."

(Perinatal_05_US)

Similarly, a provider said that: "I have noticed that [my patients in BA] are generally more nervous about coming in for their appointments... I mean, I don't disagree with them. I'm nervous to go in, too" (NSP_05_US).

\section{Objective 2. Perceived barriers to resuming in-person sessions during COVID-19}

All participant groups reported three key barriers to resuming in-person psychotherapy sessions in the future that were specific to the COVID-19 pandemic: (1) concerns about virus exposure in a hospital setting; (2) concerns about virus exposure while taking public transportation; and (3) concerns about having to wear a mask during psychotherapy sessions (see Table 3). 
Table 3

Reported barriers and facilitator to resuming in-person psychotherapy sessions during COVID-19, $n(\%)$

Key Themes

Perinatal

Provider

Stakeholder

participants

$n=23$

Participants Participants

$n=28 \quad n=18$

Barriers to resuming in-person psychotherapy sessions

Concerns about safety/virus exposure at the hospital

$17(73.9)$

$20(71.4)$

$14(77.8)$

Concerns about safety/virus exposure while taking public transit

$6(26.1)$

$7(25.0)$

$9(50.0)$

Needing to wear a mask during psychotherapy sessions

$3(13.0)$

$7(25.0)$

$9(50.0)$

\section{Facilitators to resuming in-person psychotherapy sessions}

Implementing and communicating robust safety protocols to patients

$11(47.8)$

$11(39.3)$

$13(72.2)$

Seeking alternative location to increase safety e.g. offsite, not so deep in the hospital, or in a larger room

$4(17.4)$

$9(32.1)$

$8(44.4)$

Concerns about safety and virus exposure while in the hospital

The majority of perinatal participants ( $n=17$ of $23,73.9 \%)$, providers ( $n=20$ of $28,71.4 \%$ ), and stakeholders ( $n=14$ of $18,77.8 \%$ ) expressed that future participants may be reluctant to attend in-person BA sessions due to concerns about virus exposure in a hospital setting. These participants described that pregnant and postpartum women might be particularly fearful of potentially contracting COVID-19 and transmitting it to their newborn and their families, especially at a hospital:

"If you have a new baby or are pregnant, you won't want to expose yourself [at the hospital to COVID-19] or expose your new baby. [In the SUMMIT trial women will], all be pregnant or have babies. So, it won't just be us being at risk, we'll probably have to bring our infant with us too." (Perinatal_02_ US)

"Now that we're in COVID, there's going to be a lot of anxiety... Even [if they do not bring their] kids, my general sense is that there's a fear of a hospital setting. I think that's going to be a barrier... These waiting rooms are huge, packed, full of people, [which may be a deterrent for future participants]." (NSP_06_Canada)

Some perinatal participants expressed that if they were randomized to receive in-person sessions; they would be concerned about their safety at the hospital and choose to opt-out: 
"I would be worried about COVID transmission, especially postpartum... I don't think [future participants] would be very comfortable.... I would not be very comfortable bringing my child into a session... So, if I had to go to the hospital in-person [for BA sessions], I would have chosen not to." (Perinatal_12_ Canada)

Taking public transportation

Perinatal participants ( $n=6$ of $23,26.1 \%$ ), providers ( $n=7$ of $28,25.0 \%$ ), and stakeholders ( $n=9$ of 18 , $50.0 \%$ ) described that future patients may be reluctant to attend in-person sessions because of the potential virus exposure while taking public transportation. One perinatal woman explained that:

"I'm very risk-averse... I'm not sure at what point I would feel comfortable taking the subway to come to [Canadian hospital], which is what I did before [the pandemic]... I don't know when I would feel comfortable doing that, to be honest." (Perinatal_15_ Canada)

Likewise, a U.S. provider stated that: "Transportation [would be a barrier] for clients who don't have a car... [and] having to risk exposure on the bus or the train to get to the appointment" (SP_07_US). One Canadian stakeholder highlighted that this would be a barrier especially in the winter: "We have to think about our winters [since more people take transit in the winter] and the risk of being on a very crowded transit" (Stakeholder_03_Canada).

Wearing a mask during psychotherapy sessions

Needing to wear masks during BA sessions was identified as a potential barrier by all three groups to varying degrees (perinatal participants $n=3$ of $23,13.0 \%$; providers $n=7$ of $28,25.0 \%$; and stakeholders $n$ $=9$ of $18,50.0 \%)$. Overall, participants expressed concern that wearing masks might impact the quality and experience of the BA sessions:

"[An] obstacle [is] that it's hard to imagine doing therapy in masks. It's probably going to make it so impersonal. I would not want to do that." (Perinatal_04_Canada)

"I can't really see us doing in person care until we don't have to wear masks. I don't know if [perinatal women] will feel comfortable...especially having if they have to bring their baby with them. [Providers] are used wearing [masks] all the time [but perinatal women aren't]." (NSP_04_US)

"If [the patient] is required to wear a mask in the common areas or even throughout the entire therapy, how does that feel to her? Is she okay with wearing a mask? Does that induce her anxiety?" (Stakeholder_05_Canada)

\section{Objective 3. Perceived facilitators to resuming in-person sessions during COVID-19}

Across all participant groups, the most common suggested facilitators to resuming in-person psychotherapy sessions were: (1) implementing and communicating robust safety protocols and (2) having sessions at an offsite location, not so deep within the hospital, or in larger rooms (see Table 3). 
The stakeholder group also suggested that when the SUMMIT trial intends to resume in-person sessions, recruiters for the trial should emphasize the possible benefits of in-person psychotherapy to potential participants $(n=5,27.8 \%)$ and collect further data from potential participants about their attitudes towards returning to in-person psychotherapy $(n=4,22.2 \%)$ (see Table 3 ).

Implementing and communicating robust safety protocols

Implementing and effectively communicating robust safety protocols was a facilitator suggested by 11 (47.8\%) perinatal participants, $11(39.3 \%)$ providers, and 13 (72.2\%) stakeholders. Participants suggested that information could be disseminated to patients to address patients' anxiety about coming to the hospital:

"Definitely let the patient know [what] the plans [is for] cleanliness, 'this is what we do, this is our procedure, you don't have to see anyone, you just come in, you don't have to touch anything, we're going to be 6 feet apart, there is a partition [or] Plexiglas.' Just reassuring them on that end." (Perinatal_01_US)

"We'd have to be able to provide some kind of assurance of safety in educational materials that were provided to the patients along with their SUMMIT orientation, explaining why in-person was a safe option..." (NSP_01_US)

"A way to decrease the fear and anxiety is to create a little info booklet or package that we can give to people that are consenting to in-person.... [on] the use of masks and all of the stuff... to help reduce any of those issues." (Stakeholder_07_Canada)

Conducting sessions at offsite locations, not so deep within the hospital, or in larger rooms

Another facilitator suggested by 4 (17.4\%) perinatal participants, $9(32.1 \%)$ providers, and 8 (44.4\%) stakeholders was to provider alternative locations to where sessions are delivered, such as rooms that are not so deep inside the hospitals or in larger rooms where participants can socially distance or be separated by plexiglass. For instance, participants suggested that holding sessions in a larger room could address barriers to attending in-person psychotherapy, such as concerns about having to wear masks: "Maybe [have sessions] in a larger room where you can distance even more than six feet apart" (Stakeholder_17_Canada).

Participants also suggested that conducting the BA sessions on the first floor of the hospital, or even offsite at a non-clinical location may be a facilitator for resuming in-person psychotherapy sessions:

"Instead of walking through a busy lobby, taking the elevator up, [I'd prefer] a room on the first floor as close to the door as possible [and] one person [goes in] at a time. That would be great... Walk[ing] through a healthcare facility... would be super nerve-wracking... I had to take an elevator [to see my doctor], and I was panicked for three days after." (Perinatal_23_Canada) 
"I think patients would be more interested in coming to some sort of outpatient facility or not the hospital, like a private office somewhere ... Of course, there's cost implications and cleaning and all these things." (SP_07_US)

\section{Discussion}

We examined reported barriers to accessing in-person healthcare due to COVID-19, perceived barriers that are specific to the COVID-19 pandemic, and potential facilitators to resuming in-person psychotherapy sessions during COVID-19 specific to perinatal women with symptoms of depression and anxiety in the context of the SUMMIT trial [20]. One key finding that emerged was perinatal participants and providers reported that perinatal women experienced barriers to accessing healthcare and in-person psychotherapy throughout the COVID-19 pandemic. For some perinatal participants, external barriers impacted their access to care, as many healthcare services were limited at different points of the pandemic due to changing government regulations. For others, internal barriers, namely fear and anxiety about being exposed to the virus, impacted their decisions to seek or avoid seeking healthcare services during COVID19. In addition, the majority of perinatal, provider and stakeholder participants in the current study reported significant barriers to resuming in-person psychotherapy sessions that were specific to the COVID-19 pandemic. These included include perinatal participants' concerns about virus exposure in a hospital setting, virus exposure while taking public transportation, and having to wear a mask during psychotherapy sessions.

\section{Overcoming challenges to resuming in-person psychotherapy sessions}

Perinatal participants, providers, and stakeholders all suggested two ways to facilitate resuming inperson psychotherapy sessions that are related to the aforementioned barriers. The first suggested strategy was to implement and clearly communicate robust safety protocols to study participants that is supported by research, health officials, and policy advisors across the globe, emphasizing the importance of basic safety protocols, such as requiring masks, promoting social distancing in spaces like public transportation, and employing hand hygiene when accessing public spaces [ ]. While many safety procedures are already in place at healthcare sites, patients and their providers may not be well informed about them. Thus, clearly communicating safety protocols to perinatal participants and providers will be of particular importance.

The second suggested strategy was to conduct psychotherapy not deep within the hospital setting, and also in larger-sized rooms. This suggestion may not be feasible for some studies. Nonetheless, if possible, this may help participants feel safer navigating a large hospital, where fears of being in proximity to individuals with COVID-19 may arise. Moreover, if in-person sessions are in larger rooms, then individuals may feel safer to socially distance or use plexiglass barriers if possible. 
Our findings are consistent with previous research that underscores other findings showing that patients and providers tend to feel overwhelmed when unclear messages and guidelines about health and safety exist [, ]. Studies have also shown that women and individuals with depression and anxiety are particularly risk-averse and reluctant to participate in clinical research or accessing healthcare that they perceived to be non-essential during a public health crisis, like COVID-19 [, ]. The best communication practices and strategies may include providing physical information sheets and access to a website or online PDF outlining what participants can expect when coming for in-person sessions. This information should include clearly outlined safety protocols written in straightforward terms (see Appendix $\mathrm{C}$ for a sample pamphlet for resuming in-person psychotherapy sessions). Other strategies may include providing participants with clear instructions about where to go within the hospital, having COVID-19 screening calls prior to sessions and guidelines on what to do if screens are positive, and incorporating non-punitive protocols for cancellations.

When resuming in-person psychotherapy sessions, clinical trials should be fully prepared with the necessary personal protective equipment, space, and protocols to reduce the likelihood of delays that might cause further anxiety and mistrust in future study participants. We anticipate that these recommendations can inform best practices from a transparent, patient-centered lens $\square$ when resuming not only psychotherapy sessions but other types of in-person medical interventions as well. The barriers and facilitators discussed in this study pertain to the SUMMIT trial but they can also be extrapolated to other clinical research trials and healthcare practices that are also aiming to resume in-person activities. Similarly, our findings can be used to inform practices to make in-person psychotherapy more patientcentered for perinatal participants with symptoms of depression and anxiety. This approach to implementation science aims to make health equity for perinatal women with symptoms of depression and anxiety a priority in research and in practice $[$.

COVID-19 may continue to impact future study participants' decisions to attend in-person psychotherapy, but so might the normalization and benefits of virtual care [5]. Due to the pandemic, telemedicine became a more common practice for delivering psychotherapy $[5,6]$, particularly for perinatal populations $[1,7]$. Nonetheless, in order to answer the important question of whether telemedicine psychotherapy sessions are as effective as in-person psychotherapy and for whom, creative problem solving to address potential barriers will be essential to resuming in-person sessions.

\section{Limitations and Strengths}

This study has a few limitations. First, we did not examine barriers that would exist regardless of COVID19 , such as childcare barriers. Though such barriers might exist for future participants in the study, a deeper examination of these more general barriers was outside the scope of this paper. Second, another limitation is that we did not collect demographic data for our stakeholder group. Third, given our participants were living in different jurisdictions, our findings cannot account for how variations in local government regulations impacted our participants' responses, such as the Stage two and Stage three reopening in Toronto in late summer and fall of 2020, and subsequent lockdown order at the end of 
November 2020. Future research should examine how socio-demographic factors might impact individuals' attitudes and beliefs about risks and facilitators to transitioning to in-person psychotherapy.

A key strength of this research is that we have identified key barriers and facilitators across a wide range of participant groups, including perinatal participants in the SUMMIT trial, which strengthens our findings by producing recommendations and insights that were informed directly by our participants []. This patient-centered lens is critical to the delivery of all healthcare interventions []. In addition, our systematic application of rigorous qualitative methods of barriers and facilitators [23] produced practical and clearly explained suggestions that will inform not only the SUMMIT trial, but also other outpatient treatment settings including those that cannot offer their services remotely.

\section{Conclusion}

This paper drew on multi-stakeholder, qualitative data to examine the relevant factors to resuming inperson psychotherapy session with perinatal women within the SUMMIT trial. For perinatal participants who are already experiencing depression and anxiety, reluctance to participate in in-person psychotherapy and clinical trials may be more acute. Our findings highlight critical, feasible facilitators for resuming inperson psychotherapy: the need to implement safety protocols and clearly communicate to patients and research participants the many steps being taken to maintain their health and safety.

\section{Declarations}

\section{Ethics approval and consent to participate}

Ethical approval was obtained from Clinical Trials Ontario Research Ethics Board [1895], Institutional Review Board of NorthShore University HealthSystems [EH18-129], and UNC Biomedical Institution Review Board [19-1786]. The interviewees were given/read information about the study and provided written and verbal informed consent.

\section{Consent for publication}

Not applicable.

\section{Availability of data and materials}

The dataset (which includes individual transcripts) is not publicly available due to confidentiality policies and agreements.

\section{Conflicts of Interest}

The authors declare that they have no competing interests.

\section{Funding}


The SUMMIT trial is funded as a Pragmatic Clinical Study (PCS) by the Patient-Centered

Outcomes Research Institute (PCORI), PCS-2018C1-10621. The funder was not involved in the design of the study, and is not involved in the collection, analysis, and interpretation of data, nor in writing the current manuscript. DRS is also partly funded through an Academic Scholars Award from the Department of Psychiatry at the University of Toronto.

\section{Author Contributions}

NA and DRS conceptualized this manuscript. NA wrote the initial version of the manuscript, with inputs from authors $\mathrm{SH}, \mathrm{NSM}$ and DRS, followed by contributions from SH, GS, MZ, APN, SMB, RS, SNV, NSM, and DRS. Authors SH and NA conducted the interviews and conducted the qualitative analyses. MZ and APN cleaned and analyzed the quantitative data (descriptive data). All authors have contributed to and approved the final manuscript. Author DRS is the corresponding and mentor author.

\section{Authors Information}

Interviewers information: Sabrina Hossain and Nicole Andrejek are formally trained in conducting and analyzing qualitative research methods.

\section{Acknowledgements}

We would like to sincerely thank all of our participants for participating in the focus groups and interviews for this study. We would also like to thank the larger SUMMIT research team who provided support, and have informed the development and implementation of the SUMMIT trial.

\section{References}

1. Vigod SN, Brown HK, Huang A, Fung K, Barker LC, Hussain-Shamsy N, et al. Postpartum mental illness during the COVID-19 pandemic: a population-based, repeated cross-sectional study. CMAJ. 2021 Jun 7;193(23):E835-43.

2. Basu A, Kim HH, Basaldua R, Choi KW, Charron L, Kelsall N, et al. A cross-national study of factors associated with women's perinatal mental health and wellbeing during the COVID-19 pandemic. PLoS One. 2021 Apr 21;16(4):e0249780.

3. Moverman MA, Puzzitiello RN, Pagani NR, Barnes CL, Jawa A, Menendez ME. Public Perceptions of Resuming Elective Surgery During the COVID-19 Pandemic. The Journal of Arthroplasty. 2021 Feb 1;36(2):397-402.e2.

4. Pirzada A, Awadh AA, Aleissi SA, Almeneessier AS, BaHammam AS. Reopening Sleep Medicine Services in the Conundrum of an Ongoing COVID-19 Pandemic: A Global View. Sleep Vigilance. 2020 Dec 1;4(2):73-80.

5. Monaghesh E, Hajizadeh A. The role of telehealth during COVID-19 outbreak: a systematic review based on current evidence. BMC Public Health. 2020 Aug 1;20(1):1193. 
6. Andrews G, Cuijpers P, Craske MG, McEvoy P, Titov N. Computer Therapy for the Anxiety and Depressive Disorders Is Effective, Acceptable and Practical Health Care: A Meta-Analysis. Baune BT, editor. PLoS ONE. 2010 Oct 13;5(10):e13196.

7. Lee EW, Denison FC, Hor K, Reynolds RM. Web-based interventions for prevention and treatment of perinatal mood disorders: a systematic review. BMC Pregnancy Childbirth. 2016 Dec;16(1):1-8.

8. Chen JA, Chung W-J, Young SK, Tuttle MC, Collins MB, Darghouth SL, et al. COVID-19 and telepsychiatry: Early outpatient experiences and implications for the future. General Hospital Psychiatry. 2020 Sep 1;66:89-95.

9. Dalfen A, Wasserman L, Benipal PK, Lawson A, Young B, de Oliveira C, et al. Virtual psychiatric care for perinatal depression (Virtual-PND): A pilot randomized controlled trial. Journal of Affective Disorders Reports. 2021 Apr 1;4:100085.

10. Nair U, Armfield NR, Chatfield MD, Edirippulige S. The effectiveness of telemedicine interventions to address maternal depression: A systematic review and meta-analysis. J Telemed Telecare. $2018 \mathrm{Dec}$ 1;24(10):639-50.

11. Carlbring P, Andersson G, Cuijpers P, Riper H, Hedman-Lagerlöf E. Internet-based vs. face-to-face cognitive behavior therapy for psychiatric and somatic disorders: an updated systematic review and meta-analysis. Cognitive Behaviour Therapy. 2018 Jan 2;47(1):1-18.

12. Eberly LA, Kallan MJ, Julien HM, Haynes N, Khatana SAM, Nathan AS, et al. Patient Characteristics Associated With Telemedicine Access for Primary and Specialty Ambulatory Care During the COVID19 Pandemic. JAMA Netw Open. 2020 Dec 29;3(12):e2031640.

13. Lanes A, Kuk JL, Tamim H. Prevalence and characteristics of Postpartum Depression symptomatology among Canadian women: a cross-sectional study. BMC Public Health. 2011 May $11 ; 11: 302$.

14. Bauman BL. Vital Signs: Postpartum Depressive Symptoms and Provider Discussions About Perinatal Depression - United States, 2018. MMWR Morbidity and Mortality Weekly Report. 2020. 69. Available from: https://www.cdc.gov/mmwr/volumes/69/wr/mm6919a2.htm

15. Dennis C-L, Falah-Hassani K, Shiri R. Prevalence of antenatal and postnatal anxiety: Systematic review and meta-analysis. The British Journal of Psychiatry. 2017 May 1;210(5):315-23.

16. Sockol LE, Epperson CN, Barber JP. A meta-analysis of treatments for perinatal depression. Clin Psychol Rev. 2011 Jul;31(5):839-49.

17. O’Connor E, Senger CA, Henninger ML, Coppola E, Gaynes BN. Interventions to Prevent Perinatal Depression: Evidence Report and Systematic Review for the US Preventive Services Task Force. JAMA. 2019 Feb 12;321(6):588.

18. Byatt N, Xiao RS, Dinh KH, Waring ME. Mental Health Care Use in Relation to Depressive Symptoms among Pregnant Women in the United States. Arch Womens Ment Health. 2016 Feb;19(1):187-91.

19. Salehi L, Rahimzadeh M, Molaei E, Zaheri H, Esmaelzadeh-Saeieh S. The relationship among fear and anxiety of COVID-19, pregnancy experience, and mental health disorder in pregnant women: A structural equation model. Brain and Behavior. 2020;10(11):e01835. 
20. Singla DR, Meltzer-Brody SE, Silver RK, Vigod SN, Kim JJ, La Porte LM, et al. Scaling Up Maternal Mental healthcare by Increasing access to Treatment (SUMMIT) through non-specialist providers and telemedicine: a study protocol for a non-inferiority randomized controlled trial. Trials. $2021 \mathrm{Mar}$ 5;22(1):186.

21. Eccles MP, Mittman BS. Welcome to Implementation Science. Implementation Science. 2006 Feb 22;1(1):1.

22. Wensing M, Sales A, Armstrong R, Wilson P. Implementation science in times of Covid-19. Implementation Science. 2020 Jun 8;15(1):42.

23. Shoesmith A, Hall A, Wolfenden L, Shelton RC, Powell BJ, Brown $H$, et al. Barriers and facilitators influencing the sustainment of health behaviour interventions in schools and childcare services: a systematic review. Implementation Science. 2021 Jun 12;16(1):62.

24. Singla DR, Lemberg-Pelly S, Lawson A, Zahedi N, Thomas-Jacques T, Dennis C. Implementing Psychological Interventions Through Specialist Providers and Telemedicine in High-Income Countries: Qualitative Study from a Multistakeholder Perspective. JMIR Ment Health 2020;7(8):e19271

25. Hsieh H-F, Shannon SE. Three approaches to qualitative content analysis. Qualitative health research. 2005;15(9):1277-88.

26. Starace F, Ferrara M. COVID-19 disease emergency operational instructions for Mental Health Departments issued by the Italian Society of Epidemiological Psychiatry. Epidemiology and Psychiatric Sciences. 2020; 29.

27. Schindler SE, Jicha GA, Nelson PT, Keene CD, Blennow K, Molinuevo JL, et al. Maximizing Safety in the Conduct of Alzheimer's Disease Fluid Biomarker Research in the Era of COVID-19. Journal of Alzheimer's Disease. 2020 Jan 1;76(1):27-31.

28. Coto J, Restrepo A, Cejas I, Prentiss S. The impact of COVID-19 on allied health professions. PLOS ONE. 2020 Oct 30;15(10):e0241328.

29. Devnani M. Factors Associated with the Willingness of Health Care Personnel to Work During an Influenza Public Health Emergency: An Integrative Review. Prehospital and Disaster Medicine. 2012 Dec;27(6):551-66.

30. Gobat N, Butler CC, Mollison J, Francis NA, Gal M, Harris V, et al. What the public think about participation in medical research during an influenza pandemic: an international cross-sectional survey. Public Health. 2019 Dec; 177:80-94.

31. Cardel MI, Manasse S, Krukowski RA, Ross K, Shakour R, Miller DR, et al. COVID-19 Impacts Mental Health Outcomes and Ability/Desire to Participate in Research Among Current Research Participants. Obesity. 2020;28(12):2272-81.

32. Constand MK, MacDermid JC, Dal Bello-Haas V, Law M. Scoping review of patient-centered care approaches in healthcare. BMC Health Services Research. 2014 Jun 19;14(1):271.

33. Brownson RC, Kumanyika SK, Kreuter MW, Haire-Joshu D. Implementation science should give higher priority to health equity. Implementation Science. 2021 Mar 19;16(1):28. 
34. Bombard Y, Baker GR, Orlando E, Fancott C, Bhatia P, Casalino S, et al. Engaging patients to improve quality of care: a systematic review. Implementation Science. 2018 Jul 26;13(1):98.

35. Epstein RM, Street RL. The Values and Value of Patient-Centered Care. The Annals of Family Medicine. 2011 Mar 1;9(2):100-3.

\section{Supplementary Files}

This is a list of supplementary files associated with this preprint. Click to download.

- AdditionalFile1AppendixAlnterviewguidequestions.docx

- AdditionalFile2AppendixBDemographicquestions.docx

- AdditionalFile3AppendixCBrochure.docx

- AdditionalFile4COREQChecklist.docx 